\title{
A Comparative Study on the Interaction Effect of Date of Planting, Variety and Head Diameter on the Post Harvest Quality of Broccoli Stored in Refrigerated Condition
}

\author{
Chingtham Chanbisana, A.K. Banik
}

10.18805/ajdfr.DR-1739

\begin{abstract}
Background: Broccoli [Brassica oleracea (L.) var.italica plecnk.] is a cole crop with highly perishability. It is rich in vitamin, antioxidants, anticancerous compounds and health promoting chemicals. It is harvested at tender and tight bud stage due to which the deterioration rate is high because of the high respiration rate of the broccoli head. This exotic crop is becoming popular since consumers are continuously health conscious. It has a shelf life only two days in room condition. There arise a need to retain postharvest quality of broccoli to meet the consumer demand.

Methods: Three different date of planting i.e $15^{\text {th }}$ Sept, $30^{\text {th }}$ Sept and $15^{\text {th }}$ Oct, 2014 were done in two broccoli cultivar Aishwarya and Sadhana while harvestings were done in 2015 at three different stages of head diameter i.e. $12-14 \mathrm{~cm}, 14-16 \mathrm{~cm}$ and above $16 \mathrm{~cm}$. Storage studies for biochemical changes for ascorbic acid, chlorophyll content, total soluble solids and sensory analysis were carried out in refrigerated condition.

Result: On studying the interaction effects of date of planting, variety and head diameter, it was concluded that early sown broccoli i.e $15^{\text {th }}$ September showed better quality with smaller head diameter in variety Aishwarya.

Key words: Ascorbic acid, Broccoli, Chlorophyll, Head diameter, Refrigerated, Total soluble solids.
\end{abstract}

\section{INTRODUCTION}

Broccoli (Brassica oleracea L.var.italica plecnk.) is also known as calabrese or green sprouting broccoli, Italian broccoli, asparagus broccoli, belongs to family Brassicaceae, a cool season crop and closely related to cauliflower. Broccoli is an important vegetable and a new introduction to India. It is cherished for its delicious taste, flavour and nutritive value. The crop is annual and grows to maturity in about 75 to 95 days, depending on cultivar, season and planting date. Though it is grown abundantly in the foothills of the Himalayas, in the terai regions of West Bengal, nowa-days it is gaining popularity among the vegetable growers in the Gangetic plains of West Bengal mainly due to its rich nutritional values but the information on post harvest technology aspect of the crops suitable for this region is scanty (Cantwell and Suslow, 1999).

Taking into consideration the short shelf life, high perishability and short period of availability of broccoli, the current work was undertaken to overcome the problems cited.

\section{MATERIALS AND METHODS}

Two varieties of broccoli were grown in the Horticultural Research Station, Mondouri, West Bengal at different date of sowing heads and harvested after two months in the beginning of the year 2015 at different head diameters as stated below and they were brought to the laboratory immediately in plastic crates and cooled by room condition for 3 hours. Then they were trimmed and packed in polypropylene bag and stored in as well as refrigerated condition $\left(5 \pm 1^{\circ} \mathrm{C}\right)$.
Department of Post Harvest Technology of Horticultural Crops, Bidhan Chandra Krishi Viswavidyalaya, Mohanpur, Nadia-741 252, West Bengal, India.

Corresponding Author: Chingtham Chanbisana, Department of Post Harvest Technology of Horticultural Crops, College of Horticulture, Central Agricultural University (Imphal), Thenzawl796 186, Mizoram, India. Email: chanbi.sana44@gmail.com

How to cite this article: Chanbisana, C. and Banik, A.K. (2022). A Comparative Study on the Interaction Effect of Date of Planting Variety and Head Diameter on the Post Harvest Quality of Broccoli Stored in Refrigerated Condition. Asian Journal of Dairy and Food Research. DOI: 10.18805/ajdfr.DR-1739.

Submitted: 31-05-2021 Accepted: 26-11-2021 Online: 11-02-2022

\section{Treatments}

Head diameter

$\mathrm{H}_{1}=12$ to $14 \mathrm{~cm}$ (small)

$\mathrm{H}_{2}=14$ to $16 \mathrm{~cm}$ (medium)

$\mathrm{H}_{3}=16 \mathrm{~cm}$ and above (large)

Variety

2 i.e., $V_{1}=$ Aishwarya

$$
V_{2}=\text { Sadhana }
$$

Date of sowing $\quad: \quad D_{1}=15^{\text {th }}$ September

$D_{2}=30^{\text {th }}$ September

$D_{3}=15^{\text {th }}$ October

Experimental design

Factorial CRD

Number of replication

3

Number of factor
3 
A Comparative Study on the Interaction Effect of Date of Planting, Variety and Head Diameter on the Post Harvest Quality....

\section{Observations recorded}

Physiological loss in weight $(\%)$, yellowing $(\%)$, TSS $\left({ }^{\circ} \mathrm{B}\right)$, Chlorophyll $(\mu \mathrm{g} / \mathrm{g})$, ascorbic acid $(\mathrm{mg} / 100 \mathrm{~g})$ were estimated during the period of storage by following standard procedures as described under physico chemical analysis in refrigerated condition (5 days interval).

\section{Physical and chemical analysis}

\section{Physiological loss in weight (PLW)}

PLW was calculated as total weight loss \%, based on the initial weight (before storage) and weight loss at the day of sampling during storage (Nath et al., 2011).

$$
\text { PLW }=\frac{\text { Initial weight }- \text { Final weight }}{\text { Initial weight }} \times 100
$$

\section{Ascorbic acid}

Volumetric method of ascorbic acid determination (2,6Dichlorophenol-Indophenol Visual Titration Method) was done using 2, 6-dichlorophenol indophenols which gets reduced to a colourlessleuco-base by ascorbic acid and the ascorbic acid gets oxidised to dehydroascorbic acid. Though the dye is a blue coloured compound, the end point is the appearance of pink colour. The dye is pink coloured in acid medium (Ranganna, 1986).

Ascorbic acid $(\mathrm{mg} / 100 \mathrm{~g})=$

Titre value $\times$ Dye factor $\times$ Volume made up

Aliquot of extract taken for estimation $\times \mathrm{Wt}$. $\times 100$

or vol: of the sample taken for estimation

\section{Chlorophyll}

Total chlorophyll content was determined by spectrophotometric method (A.O.A.C,1990). A known amount of tissue sample by weight is taken and chlorophyll is extracted in $80 \%$ acetone until the residue has no more green colour. The filtrate or supernatant is made upto known volume with $80 \%$ acetone and the Optical Density (OD) value is then measured through $660 \mathrm{~nm}$ and $642.5 \mathrm{~nm}$ wavelength in a colorimeter against blank. Using the adsorption coefficients, the amount of chlorophyll is calculated as follows:

Total chlorophyll $(\mathrm{a}+\mathrm{b}), \mu \mathrm{g} / \mathrm{ml}=(7.12 \times \mathrm{OD}$ at $660 \mathrm{~nm})+$ $(16.8 \times \mathrm{OD}$ at $642.5 \mathrm{~nm})$

\section{Total soluble solids (TSS) ${ }^{\circ}$ Brix}

TSS was determined by Hand Refractometer (Erma Hand Refractrometer I.S.O 2173), by principle of total reflection which was calibrated at $0^{\circ} \mathrm{B}$ is checked before use by placing few drops of distilled water on the prism in the specimen chamber of the refractrometer. The refractive index is the ratio of the speed of light in vacuum to its speed in a substance and is used as a measure of concentration of solutes in solution (Peris, 2004).

\section{Yellowing percentage}

Whole green broccoli head is divided into four parts out of 100 per cent. The area which becomes yellow is recorded visually according to the four parts divided. There were two conditions of yellowing, one where there was gradual yellowing while the other was with the patchy development of colour.

\section{Marketability (\%)}

The marketability of broccoli was determined on the basis of colour, texture or crispiness, flavour and defects like yellowing, browning and decay and expressed in percentage (\%). Upto an amount of $50 \%$ was considered to be marketable.

\section{RESULTS AND DISCUSSION \\ Physiological loss in weight (PLW) \%}

PLW increased with the advance of storage days in Table 1. Higher loss in weight was observed in late sown crop $\left(D_{3}\right)$ increasing from 2.17 to $6.69 \%$ throughout the storage period while the lowest loss in weight was noted in early sown crop $\left(D_{1}\right)$ while minimum loss in weight was prominent in Aishwarya $\left(V_{1}\right)$ ranging from 1.43 to $5.08 \%$ from 5 to 15 days after storage. From the statistically analysed data in Table 1, the least value of loss in weight throughout the storage was observed in small size diameter of 12 to $14 \mathrm{~cm}\left(\mathrm{H}_{1}\right)$ ranging from 1.55 to $5.11 \%$ throughout the storage study.

Interaction effect of date of sowing, variety and head diameter indicated significant variation in physiological loss in weight of broccoli during storage period. Lowest loss in weight ( 0.535 days after storage) was observed in Sadhana sown at $15^{\text {th }}$ September with 12 to $14 \mathrm{~cm}$ head $\left(\mathrm{D}_{1} \mathrm{~V}_{2} \mathrm{H}_{1}\right)$. Weight losses were between 0.6 and $1.6 \%$ of original fresh weight supported by the findings of Jacobsson et al. (2004). Early shown crops had better PLW which may be attributed to the firm texture and good shape favoured by the good weather for growing while high temperature in the late sown crops leads to bit loose heads. Nath et al. (2011) showed results on PLW of broccoli florets indicated that weight loss was gradually increased. The maximum shelf life (19.67 days) of broccoli was recorded at $4^{\circ} \mathrm{C}$ (Roni et al., 2014) while our current research was emphasized for extension of shelf life through protective covering for 15 days at $5^{\circ} \mathrm{C}$.

\section{Marketability (\%)}

Marketability (\%) was high in mid sown broccoli at $30^{\text {th }}$ September compared to other date of sowing as shown in Table 1. Sadhana showed higher marketability than Aishwarya though both have fair marketability \%. Small head diameter also gave higher marketability compared to other larger head diameter. Interaction effect of date of sowing, variety and head diameter gave highest marketability in Sadhana sown at $15^{\text {th }}$ September and harvested at small head diameter of 12 to $14 \mathrm{~cm}$. Weight loss in crop lead not only to physical weight loss but also leads to change in appearance, color, texture of produce whch ultimately decreases the market value as well as consumer preference (Pragya et al., 2020).

\section{TSS ( $\left.{ }^{\circ} \mathrm{B}\right)$}

It was observed in Table 2 that TSS was maximum and constant $\left(7.77^{\circ} \mathrm{B}\right.$ in 5,10 and 15 days after storage 
respectively) in early sown crop at $15^{\text {th }}$ September $\left(D_{1}\right)$. Among the varieties considered, Aishwarya $\left(V_{1}\right)$ exhibited higher TSS (7.82. 7.36 and $7.07^{\circ} \mathrm{B}$ on 5,10 and 15 days after sowing respectively) compared to Sadhana $\left(\mathrm{V}_{2}\right)$. From Table 2, it was shown that among the various head diameters, medium size broccoli with 14 to $16 \mathrm{~cm}$ diameter resulted higher TSS with $7.57,7.38$ and $7.24^{\circ} \mathrm{B}$ on 5,10 and 15 days after storage respectively.

Maximum TSS during storage was observed in Aishwarya sown early at $15^{\text {th }}$ September with medium head diameter of 12 to $14 \mathrm{~cm}\left(\mathrm{D}_{1} \mathrm{~V}_{1} \mathrm{H}_{2}\right)$. Loss in soluble solids during storage may be correlated with the finding of $\mathrm{Ihl}$ et al. (2001) where the soluble solid content decreased when sugar snap peas were stored at $5^{\circ} \mathrm{C}$ in sealed packages.
Increasing trend in TSS in early sown crops may be supported by the findings of Mahfuzah et al. (2013) in packed strawberry while Galvao et al. (2008) reported that up to the first week of storage, marked reduction in soluble starch contents was observed in packed broccoli.

\section{Ascorbic acid $(\mathrm{mg} / \mathrm{g})$}

Table 3 showed the maximum content in late sown crop at $15^{\text {th }}$ October $\left(D_{3}\right)$ with $124.56,109.86$ and $96.75 \mathrm{mg} / 100 \mathrm{~g}$ during storage period.

There was significant effect of variety on ascorbic acid content during the storage. Aishwarya $\left(\mathrm{V}_{1}\right)$ exhibited higher values $(140.52,128.71,110.49$ and $93.53 \mathrm{mg} / 100)$ from harvest to 15 days after storage respectively.

Table 1: Effect of head diameter, variety, date of sowing and their interaction on physiological loss in weight (\%) in refrigerated condition

\begin{tabular}{|c|c|c|c|c|c|c|}
\hline \multirow[t]{2}{*}{ Date of sowing } & \multirow[t]{2}{*}{ Variety } & \multirow[t]{2}{*}{ Head diameter $(\mathrm{cm})$} & \multicolumn{3}{|c|}{ Days in storage } & \multirow{2}{*}{$\begin{array}{l}\text { Market-abilts } \\
\quad(\%) \text { on } 15^{t}\end{array}$} \\
\hline & & & 5 & 10 & 15 & \\
\hline \multicolumn{7}{|c|}{ Effect of date of sowing } \\
\hline $15^{\text {th }}$ Sept $\left(D_{1}\right)$ & & & $1.45(1.55)$ & $3.44(2.09)$ & $4.99(2.43)$ & 67.50 \\
\hline $30^{\text {th }}$ Sept $\left(D_{2}\right)$ & & & $2.05(1.72)$ & $3.85(2.18)$ & $5.92(2.61)$ & 86.39 \\
\hline $15^{\text {th }}$ Oct $\left(D_{3}\right)$ & & & $2.17(1.77)$ & $4.45(2.31)$ & $6.69(2.76)$ & 83.89 \\
\hline Sem \pm & & & 0.06 & 0.02 & 0.02 & 0.66 \\
\hline$C D(P=0.05)$ & & & 0.03 & 0.20 & 0.07 & 1.89 \\
\hline \multicolumn{7}{|l|}{ Effect of variety } \\
\hline & Aishwarya $\left(\mathrm{V}_{1}\right)$ & & $1.43(1.54)$ & $2.94(1.97)$ & $5.08(2.44)$ & 77.22 \\
\hline & Sadhana $\left(\mathrm{V}_{2}\right)$ & & $2.35(1.82)$ & $4.88(2.42)$ & $6.65(2.76)$ & 81.30 \\
\hline Sem \pm & & & 0.05 & 0.02 & 0.02 & 0.54 \\
\hline$C D(P=0.05)$ & & & 0.02 & 0.07 & 0.06 & 1.55 \\
\hline \multicolumn{7}{|c|}{ Effect of head diameter } \\
\hline & 12 to $14\left(\mathrm{H}_{1}\right)$ & & $1.55(1.59)$ & $3.23(2.04)$ & $5.11(2.44)$ & 86.67 \\
\hline & 14 to $16\left(\mathrm{H}_{2}\right)$ & & $1.60(1.58)$ & $3.68(2.14)$ & $6.13(2.66)$ & 78.33 \\
\hline & Above $16\left(\mathrm{H}_{3}\right)$ & & $2.53(1.89)$ & $4.83(2.39)$ & $6.36(2.70)$ & 72.78 \\
\hline Sem \pm & & & 0.06 & 0.02 & 0.02 & 0.66 \\
\hline$C D(P=0.05)$ & & & 0.03 & 0.08 & 0.08 & 1.89 \\
\hline \multicolumn{7}{|c|}{ Effect of interaction } \\
\hline & & 12 to $14\left(\mathrm{H}_{1}\right)$ & $1.97(1.72)$ & $4.20(2.28)$ & $6.67(2.77)$ & 81.67 \\
\hline \multirow[t]{3}{*}{$15^{\text {th }}$ Sept $\left(D_{1}\right)$} & Aishwarya $\left(\mathrm{V}_{1}\right)$ & 14 to $16\left(\mathrm{H}_{2}\right)$ & $1.86(1.69)$ & $4.28(2.29)$ & $5.41(2.53)$ & 71.67 \\
\hline & & Above $16\left(\mathrm{H}_{3}\right)$ & $1.20(1.48)$ & $4.60(2.35)$ & $4.87(2.42)$ & 56.67 \\
\hline & & 12 to $14\left(\mathrm{H}_{1}\right)$ & $1.00(1.41)$ & $1.70(1.64)$ & $2.70(1.92)$ & 88.33 \\
\hline \multirow[t]{3}{*}{$15^{\text {th }}$ Sept $\left(D_{1}\right)$} & Sadhana $\left(V_{2}\right)$ & 14 to $16\left(\mathrm{H}_{2}\right)$ & $0.53(1.23)$ & $2.83(1.93)$ & $4.17(2.26)$ & 63.33 \\
\hline & & Above $16\left(\mathrm{H}_{3}\right)$ & $2.13(1.77)$ & $3.03(2.00)$ & $6.17(2.67)$ & 43.33 \\
\hline & & 12 to $14\left(\mathrm{H}_{1}\right)$ & $2.13(1.77)$ & $4.73(2.39)$ & $7.80(2.97)$ & 83.33 \\
\hline \multirow[t]{3}{*}{$30^{\text {th }}$ Sept $\left(D_{2}\right)$} & Aishwarya $\left(\mathrm{V}_{1}\right)$ & 14 to $16\left(\mathrm{H}_{2}\right)$ & $2.46(1.86)$ & $4.22(2.28)$ & $6.15(2.67)$ & 78.33 \\
\hline & & Above $16\left(\mathrm{H}_{3}\right)$ & $3.33(2.08)$ & $6.17(2.67)$ & $7.47(2.91)$ & 90.00 \\
\hline & & 12 to $14\left(\mathrm{H}_{1}\right)$ & $0.73(1.32)$ & $1.70(1.64)$ & $2.87(1.96)$ & 90.00 \\
\hline \multirow[t]{3}{*}{$30^{\text {th }}$ Sept $\left(D_{2}\right)$} & Sadhana $\left(V_{2}\right)$ & 14 to $16\left(\mathrm{H}_{2}\right)$ & $0.57(1.25)$ & $2.77(1.94)$ & $6.03(2.65)$ & 90.00 \\
\hline & & Above $16\left(\mathrm{H}_{3}\right)$ & $3.10(2.03)$ & $3.50(2.12)$ & $5.17(2.48)$ & 86.67 \\
\hline & & 12 to $14\left(\mathrm{H}_{1}\right)$ & $1.97(1.72)$ & $4.10(2.26)$ & $6.40(2.72)$ & 86.67 \\
\hline \multirow[t]{3}{*}{$15^{\text {th }}$ Oct $\left(D_{3}\right)$} & Aishwarya $\left(V_{1}\right)$ & 14 to $16\left(\mathrm{H}_{2}\right)$ & $3.35(2.08)$ & $5.76(2.60)$ & $8.46(3.08)$ & 76.67 \\
\hline & & Above $16\left(\mathrm{H}_{3}\right)$ & $2.90(1.97)$ & $5.90(2.63)$ & $6.67(2.76)$ & 70.00 \\
\hline & & 12 to $14\left(\mathrm{H}_{1}\right)$ & $1.50(1.58)$ & $2.97(1.99)$ & $4.23(2.28)$ & 90.00 \\
\hline \multirow[t]{2}{*}{$15^{\text {th }}$ Oct $\left(D_{3}\right)$} & Sadhana $\left(V_{2}\right)$ & 14 to $16\left(\mathrm{H}_{2}\right)$ & $0.83(1.35)$ & $2.23(1.79)$ & $3.53(2.75)$ & 90.00 \\
\hline & & Above $16\left(\mathrm{H}_{3}\right)$ & $2.50(1.87)$ & $5.77(2.60)$ & $7.83(2.97)$ & 90.00 \\
\hline Sem \pm & & & 0.14 & 0.06 & 0.06 & 1.62 \\
\hline$C D(P=0.05)$ & & & 0.07 & 0.19 & 0.19 & 4.65 \\
\hline
\end{tabular}


Head diameter showed significant effect on ascorbic acid during the storage study. Maximum ascorbic acid contents vary from 136.06 to $98.28 \mathrm{mg} / 100$ throughout the storage period in early sown crops at $15^{\text {th }}$ September $\left(H_{1}\right)$. Steady decrease in amount of ascorbic acid with storage period was evident in mushroom packed with modified atmosphere packaging unit after blanching followed by treatment with $2.5 \%$ calcium chloride, $3 \%$ citric acid and $0.1 \%$ sorbitol (Pallavi et al., 2020).

Considering the interaction effect in Table 3 , the maximum values were recorded in Aishwarya sown at $15^{\text {th }}$ September with 12 to $14 \mathrm{~cm}$ head $\left(D_{1} V_{1} H_{1}\right)$. Broccoli sown at $15^{\text {th }}$ September $\left(D_{1}\right)$ had the overall high amount of ascorbic acid during the period of study. Present study revealed that drastic change in ascorbic acid content has been reduced giving appreciable amount of ascorbic acid at the end of storage in refrigerated condition and according to studies conducted on different cultivars with distinct conditions, MAP was significantly effective to prevent drastic ascorbic acid loss in broccoli (Serrano et al., 2006, Carvalho and Clemente, 2004).

\section{Chlorophyll $(\mu \mathrm{g} / \mathrm{g})$}

From the data analysed in Table 4, it was clear that date of sowing gave significant effect on chlorophyll content of broccoli during the storage period. Maximum chlorophyll content was observed in mid sown crop at $30^{\text {th }}$ September $\left(D_{2}\right)$ with 312.69 , 305.53 and $298.52 \mu \mathrm{g} / \mathrm{g}$ on 5, 10 and 15days after storage

Table 2: Effect of head diameter, variety, date of sowing and their interaction on total soluble solids ( $\left.{ }^{\circ} \mathrm{B}\right)$ content in refrigerated condition.

\begin{tabular}{|c|c|c|c|c|c|c|}
\hline \multirow[t]{2}{*}{ Date of sowing } & \multirow[t]{2}{*}{ Variety } & \multirow[t]{2}{*}{ Head diameter $(\mathrm{cm})$} & \multicolumn{4}{|c|}{ Days in storage } \\
\hline & & & At harvest & 5 & 10 & 15 \\
\hline \multicolumn{7}{|c|}{ Effect of date of sowing } \\
\hline $15^{\text {th }}$ Sept $\left(D_{1}\right)$ & & & 7.73 & 7.77 & 7.77 & 7.77 \\
\hline $30^{\text {th }}$ Sept $\left(D_{2}\right)$ & & & 7.38 & 7.16 & 7.27 & 6.84 \\
\hline $15^{\text {th }}$ Oct $\left(D_{3}\right)$ & & & 7.69 & 7.79 & 6.98 & 6.43 \\
\hline Sem \pm & & & 0.12 & 0.04 & 0.07 & 0.06 \\
\hline$C D(P=0.05)$ & & & NS & 0.14 & NS & 0.19 \\
\hline \multicolumn{7}{|l|}{ Effect of variety } \\
\hline & Aishwarya $\left(V_{1}\right)$ & & 7.88 & 7.82 & 7.36 & 7.07 \\
\hline & Sadhana $\left(\mathrm{V}_{2}\right)$ & & 7.32 & 7.33 & 7.31 & 6.96 \\
\hline Sem \pm & & & 0.10 & 0.03 & 0.05 & 0.05 \\
\hline$C D(P=0.05)$ & & & 0.29 & 0.11 & NS & NS \\
\hline \multicolumn{7}{|c|}{ Effect of head diameter } \\
\hline & & 12 to $14\left(\mathrm{H}_{1}\right)$ & 7.62 & 7.57 & 7.30 & 7.12 \\
\hline & & 14 to $16\left(\mathrm{H}_{2}\right)$ & 7.61 & 7.57 & 7.38 & 7.24 \\
\hline & & Above $16\left(\mathrm{H}_{3}\right)$ & 7.57 & 7.62 & 7.32 & 6.68 \\
\hline Sem \pm & & & 0.12 & 0.04 & 0.07 & 0.06 \\
\hline$C D(P=0.05)$ & & & NS & NS & NS & 0.19 \\
\hline \multicolumn{7}{|c|}{ Effect of interaction } \\
\hline & & 12 to $14\left(\mathrm{H}_{1}\right)$ & 8.16 & 7.30 & 7.27 & 6.80 \\
\hline \multirow[t]{3}{*}{$15^{\text {th }}$ Sept $\left(D_{1}\right)$} & Aishwarya $\left(\mathrm{V}_{1}\right)$ & 14 to $16\left(\mathrm{H}_{2}\right)$ & 8.50 & 9.03 & 8.53 & 10.13 \\
\hline & & Above $16\left(\mathrm{H}_{3}\right)$ & 8.57 & 9.07 & 9.10 & 7.50 \\
\hline & & 12 to $14\left(\mathrm{H}_{1}\right)$ & 7.03 & 7.07 & 7.57 & 7.93 \\
\hline \multirow[t]{3}{*}{$15^{\text {th }}$ Sept $\left(D_{1}\right)$} & Sadhana $\left(\mathrm{V}_{2}\right)$ & 14 to $16\left(\mathrm{H}_{2}\right)$ & 7.10 & 7.20 & 7.13 & 7.20 \\
\hline & & Above $16\left(\mathrm{H}_{3}\right)$ & 7.03 & 6.97 & 7.00 & 7.07 \\
\hline & & 12 to $14\left(\mathrm{H}_{1}\right)$ & 7.43 & 7.23 & 7.20 & 7.20 \\
\hline \multirow[t]{3}{*}{$30^{\text {th }}$ Sept $\left(D_{2}\right)$} & Aishwarya $\left(\mathrm{V}_{1}\right)$ & 14 to $16\left(\mathrm{H}_{2}\right)$ & 7.30 & 6.73 & 6.97 & 7.00 \\
\hline & & Above $16\left(\mathrm{H}_{3}\right)$ & 7.30 & 6.37 & 6.50 & 6.47 \\
\hline & & 12 to $14\left(\mathrm{H}_{1}\right)$ & 7.66 & 7.83 & 7.10 & 6.90 \\
\hline \multirow[t]{3}{*}{$30^{\text {th }}$ Sept $\left(D_{2}\right)$} & Sadhana $\left(V_{2}\right)$ & 14 to $16\left(\mathrm{H}_{2}\right)$ & 7.43 & 6.87 & 7.83 & 6.87 \\
\hline & & Above $16\left(\mathrm{H}_{3}\right)$ & 7.13 & 7.93 & 8.00 & 6.60 \\
\hline & & 12 to $14\left(\mathrm{H}_{1}\right)$ & 7.93 & 8.60 & 7.50 & 6.93 \\
\hline \multirow[t]{3}{*}{$15^{\text {th }}$ Oct $\left(D_{3}\right)$} & Aishwarya $\left(\mathrm{V}_{1}\right)$ & 14 to $16\left(\mathrm{H}_{2}\right)$ & 7.83 & 8.03 & 6.83 & 5.83 \\
\hline & & Above $16\left(\mathrm{H}_{3}\right)$ & 7.87 & 8.00 & 6.33 & 5.80 \\
\hline & & 12 to $14\left(\mathrm{H}_{1}\right)$ & 7.50 & 7.40 & 7.17 & 6.97 \\
\hline \multirow[t]{2}{*}{$15^{\text {th }}$ Oct $\left(D_{3}\right)$} & Sadhana $\left(\mathrm{V}_{2}\right)$ & 14 to $16\left(\mathrm{H}_{2}\right)$ & 7.46 & 7.33 & 7.00 & 6.43 \\
\hline & & Above $16\left(\mathrm{H}_{3}\right)$ & 7.53 & 7.37 & 7.00 & 6.63 \\
\hline Sem \pm & & & 0.30 & 0.11 & 0.17 & 0.16 \\
\hline$C D(P=0.05)$ & & & NS & 0.33 & 0.50 & 0.47 \\
\hline
\end{tabular}


while minimum chlorophyll contents of 275.33 to $242.22 \mu \mathrm{g} /$ $\mathrm{g}$ during the storage period were noted in early sown crop at $15^{\text {th }}$ September $\left(D_{1}\right)$.

Variety gave significant effect on chlorophyll content of broccoli during storage. Aishwarya $\left(V_{1}\right)$ showed maximum values from 298.77 to $276.84 \mu \mathrm{g} / \mathrm{g}$ during the storage period while Sadhana $\left(\mathrm{V}_{2}\right)$ showed lower values from 289.48 to $258.41 \mu \mathrm{g} / \mathrm{g}$ during the period of the storage study.

It is revealed from Table 10 head diameter gave significant variation in chlorophyll content during the storage study. Large size broccoli gave higher chlorophyll contents $(298.77,281.16$ and $276.84 \mu \mathrm{g} / \mathrm{g}$ on 5,10 and 15 days after storage respectively) while small size broccoli $\left(\mathrm{H}_{1}\right)$ gave minimum chlorophyll contents $(287.73$,
265.87 and $251.05 \mu \mathrm{g} / \mathrm{g}$ on 5,10 and 15 days after storage respectively).

Interaction effect of date of sowing, variety and head diameter gave significant effect on the chlorophyll contents of broccoli during the storage period resulting in maximum chlorophyll content $(323.66 \mu \mathrm{g} / \mathrm{g})$ during the storage period in Aishwarya sown at $30^{\text {th }}$ September with head diameter 14 to $16 \mathrm{~cm}\left(\mathrm{D}_{2} \mathrm{~V}_{1} \mathrm{H}_{2}\right)$ while minimum chlorophyll contents $(173.3 \mu \mathrm{g} / \mathrm{g})$ were recorded in Aishwarya sown at $15^{\text {th }}$ October with head diameter 12 to $14 \mathrm{~cm}\left(\mathrm{D}_{3} \mathrm{~V}_{1} \mathrm{H}_{1}\right)$.

Maximum chlorophyll content was found in broccoli sown at $30^{\text {th }}$ September, $D_{2}$ for the period of storage study while variety Aishwarya showed higher chlorophyll content than Sadhana. Since freshly harvested broccoli is composed

Table 3: Effect of head diameter, variety, date of sowing and their interaction on ascorbic acid (mg/100 $\mathrm{g})$ in refrigerated condition.

\begin{tabular}{|c|c|c|c|c|c|c|}
\hline \multirow[t]{2}{*}{ Date of sowing } & \multirow[t]{2}{*}{ Variety } & \multirow[t]{2}{*}{ Head diameter $(\mathrm{cm})$} & \multicolumn{3}{|c|}{ Days in storage } & \multirow[b]{2}{*}{15} \\
\hline & & & At harvest & 5 & 10 & \\
\hline \multicolumn{7}{|c|}{ Effect of date of sowing } \\
\hline $15^{\text {th }}$ Sept $\left(D_{1}\right)$ & & & 134.00 & 118.78 & 111.17 & 96.00 \\
\hline $30^{\text {th }}$ Sept $\left(D_{2}\right)$ & & & 136.944 & 124.11 & 107.39 & 77.51 \\
\hline $15^{\text {th }}$ Oct $\left(D_{3}\right)$ & & & 134.67 & 124.56 & 109.86 & 96.75 \\
\hline Sem \pm & & & 1.09 & 1.09 & 1.26 & 1.01 \\
\hline$C D(P=0.05)$ & & & NS & 3.13 & 3.64 & 2.92 \\
\hline \multicolumn{7}{|l|}{ Effect of variety } \\
\hline & Aishwarya $\left(\mathrm{V}_{1}\right)$ & & 140.52 & 128.71 & 110.49 & 93.53 \\
\hline & Sadhana $\left(\mathrm{V}_{2}\right)$ & & 129.89 & 116.26 & 108.46 & 86.65 \\
\hline Sem \pm & & & 0.89 & 0.88 & 1.03 & 0.83 \\
\hline$C D(P=0.05)$ & & & 2.57 & 2.56 & NS & 2.38 \\
\hline \multicolumn{7}{|c|}{ Effect of head diameter } \\
\hline & & 12 to $14\left(\mathrm{H}_{1}\right)$ & 136.06 & 134.944 & 121.39 & 98.28 \\
\hline & & 14 to $16\left(\mathrm{H}_{2}\right)$ & 134.56 & 110.336 & 105.31 & 87.01 \\
\hline & & Above $16\left(\mathrm{H}_{3}\right)$ & 135 & 122.17 & 101.72 & 84.98 \\
\hline Sem \pm & & & 1.09 & 1.09 & 1.26 & 1.01 \\
\hline$C D(P=0.05)$ & & & NS & 3.14 & 3.644 & 2.92 \\
\hline \multicolumn{7}{|c|}{ Effect of interaction } \\
\hline & & 12 to $14\left(\mathrm{H}_{1}\right)$ & 142.33 & 142.67 & 142.33 & 128.67 \\
\hline \multirow[t]{3}{*}{$15^{\text {th }}$ Sept $\left(D_{1}\right)$} & Aishwarya $\left(\mathrm{V}_{1}\right)$ & 14 to $16\left(\mathrm{H}_{2}\right)$ & 142.67 & 134.67 & 121.00 & 92.33 \\
\hline & & Above $16\left(\mathrm{H}_{3}\right)$ & 142.33 & 111.33 & 89.33 & 85.00 \\
\hline & & 12 to $14\left(\mathrm{H}_{1}\right)$ & 125.00 & 126.00 & 124.00 & 108.33 \\
\hline \multirow[t]{3}{*}{$15^{\text {th }}$ Sept $\left(D_{1}\right)$} & Sadhana $\left(V_{2}\right)$ & 14 to $16\left(\mathrm{H}_{2}\right)$ & 142.33 & 71.33 & 64.00 & 50 \\
\hline & & Above $16\left(\mathrm{H}_{3}\right)$ & 127.667 & 126.67 & 126.00 & 111.67 \\
\hline & & 12 to $14\left(\mathrm{H}_{1}\right)$ & 141.67 & 139.33 & 97.33 & 80.48 \\
\hline \multirow[t]{3}{*}{$30^{\text {th }}$ Sept $\left(D_{2}\right)$} & Aishwarya $\left(\mathrm{V}_{1}\right)$ & 14 to $16\left(\mathrm{H}_{2}\right)$ & 134.00 & 113.68 & 98.33 & 95.58 \\
\hline & & Above $16\left(\mathrm{H}_{3}\right)$ & 135.00 & 123.33 & 100.67 & 55.02 \\
\hline & & 12 to $14\left(\mathrm{H}_{1}\right)$ & 143.33 & 141.00 & 141.33 & 83.0 \\
\hline \multirow[t]{3}{*}{$30^{\text {th }}$ Sept $\left(D_{2}\right)$} & Sadhana $\left(V_{2}\right)$ & 14 to $16\left(\mathrm{H}_{2}\right)$ & 135.00 & 111.00 & 111.67 & 88.33 \\
\hline & & Above $16\left(\mathrm{H}_{3}\right)$ & 133.33 & 116.33 & 95.00 & 62.67 \\
\hline & & 12 to $14\left(\mathrm{H}_{1}\right)$ & 138.33 & 138.33 & 115.00 & 93.33 \\
\hline \multirow[t]{3}{*}{$15^{\text {th }}$ Oct $\left(D_{3}\right)$} & Aishwarya $\left(\mathrm{V}_{1}\right)$ & 14 to $16\left(\mathrm{H}_{2}\right)$ & 143.33 & 113.33 & 106.67 & 106.67 \\
\hline & & Above $16\left(\mathrm{H}_{3}\right)$ & 145.00 & 141.67 & 123.33 & 104.67 \\
\hline & & 12 to $14\left(\mathrm{H}_{1}\right)$ & 125.67 & 122.33 & 108.33 & 95.83 \\
\hline \multirow[t]{2}{*}{$15^{\text {th }}$ Oct $\left(D_{3}\right)$} & Sadhana $\left(V_{2}\right)$ & 14 to $16\left(\mathrm{H}_{2}\right)$ & 129.00 & 118.00 & 108.33 & 89.167 \\
\hline & & Above $16\left(\mathrm{H}_{3}\right)$ & 126.67 & 113.67 & 97.50 & 90.83 \\
\hline Sem \pm & & & 2.68 & 2.67 & 3.11 & 2.49 \\
\hline$C D(P=0.05)$ & & & NS & 7.67 & 8.92 & 7.15 \\
\hline
\end{tabular}


by immature tissues, the inflorescence head shows rapid senescence and chlorophyll degradation (Clarke et al., 1994; Corcuff et al.1996). Decreasing trend of chlorophyll was supported by Nath et al. (2011) in broccoli florets which underwent chlorophyll degradation and a lower temperature resulted in less deterioration during post-harvest storage. The decrease in chlorophyll was supported by Rai et al. (2008) while Starzyńska et al. (2003) reported that chlorophyll was degraded at a slower rate in the broccoli stored at $0^{\circ} \mathrm{C}$.

\section{Yellowing percentage}

Data presented in Table 5 indicated increasing yellowing \% with the progress in storage period. Maximum yellowing of $14 \%$ was recorded in late sown broccoli at $15^{\text {th }}$ October $\left(D_{3}\right)$ while minimum yellowing in early sown crop $30^{\text {th }}$ September $\left(D_{1}\right)$ ranging from 0.83 to $1.00 \%$ from day 5 to 15 days after storage.

It was clear from Table 5 that among the two varieties, Aishwarya $\left(\mathrm{V}_{1}\right)$ had higher amount of yellowing $(0.55$ to $8.04 \%$ throughout the storage period) during the storage period while larger broccoli head gave highest amount on yellowing on day 15 (16.89\%) throughout the storage period. Interaction among date of sowing and head diameter gave significant effect on yellowing \% of broccoli during the storage period with minimum was observed in Aishwarya sown at $15^{\text {th }}$ October with 12 to $14 \mathrm{~cm}$ head,

Table 4: Effect of head diameter, variety, date of sowing and their interaction on chlorophyll $(\mu \mathrm{g} / \mathrm{g})$ in refrigerated condition.

\begin{tabular}{|c|c|c|c|c|c|c|}
\hline & \multirow[t]{2}{*}{ Variety } & \multirow[t]{2}{*}{ Head diameter $(\mathrm{cm})$} & \multicolumn{3}{|c|}{ Days in storage } & \multirow[b]{2}{*}{15} \\
\hline & & & At harvest & 5 & 10 & \\
\hline \multicolumn{7}{|c|}{ Effect of date of sowing } \\
\hline $15^{\text {th }}$ Sept $\left(D_{1}\right)$ & & & 295.94 & 275.33 & 262.78 & 242.22 \\
\hline $30^{\text {th }}$ Sept $\left(D_{2}\right)$ & & & 314.84 & 312.69 & 305.53 & 298.52 \\
\hline $15^{\text {th }}$ Oct $\left(D_{3}\right)$ & & & 302.33 & 294.35 & 245.63 & 262.13 \\
\hline Sem \pm & & & 1.11 & 1.35 & 1.23 & 1.13 \\
\hline$C D(P=0.05)$ & & & NS & 3.90 & 3.53 & 3.25 \\
\hline \multicolumn{7}{|c|}{ Effect of variety } \\
\hline & Aishwarya $\left(\mathrm{V}_{1}\right)$ & & 307.67 & 298.77 & 281.16 & 276.84 \\
\hline & Sadhana $\left(\mathrm{V}_{2}\right)$ & & 301.07 & 289.48 & 261.47 & 258.41 \\
\hline Sem \pm & & & 0.90 & 1.10 & 1.00 & 0.92 \\
\hline$C D(P=0.05)$ & & & 2.61 & 3.17 & 2.88 & 2.65 \\
\hline \multicolumn{7}{|c|}{ Effect of head diameter } \\
\hline & & 12 to $14\left(\mathrm{H}_{1}\right)$ & 303.73 & 287.73 & 265.87 & 251.05 \\
\hline & & 14 to $16\left(\mathrm{H}_{2}\right)$ & 305.01 & 297.53 & 280.88 & 265.50 \\
\hline & & Above $16\left(\mathrm{H}_{3}\right)$ & 304.36 & 297.12 & 267.19 & 386.32 \\
\hline Sem \pm & & & 1.11 & 1.35 & 1.23 & 1.13 \\
\hline$C D(P=0.05)$ & & & NS & 3.89 & 3.53 & 3.25 \\
\hline \multicolumn{7}{|c|}{ Effect of interaction } \\
\hline & & 12 to $14\left(\mathrm{H}_{1}\right)$ & 292.33 & 272.33 & 265.66 & 254.66 \\
\hline \multirow{3}{*}{$15^{\text {th }}$ Sept $\left(D_{1}\right)$} & Aishwarya $\left(\mathrm{V}_{1}\right)$ & 14 to $16\left(\mathrm{H}_{2}\right)$ & 293.00 & 271.00 & 255.00 & 224.33 \\
\hline & & Above $16\left(\mathrm{H}_{3}\right)$ & 292.33 & 254.00 & 235.33 & 212.33 \\
\hline & & 12 to $14\left(\mathrm{H}_{1}\right)$ & 297.66 & 292.00 & 287.00 & 281.33 \\
\hline \multirow[t]{3}{*}{$15^{\text {th }}$ Sept $\left(D_{1}\right)$} & Sadhana $\left(V_{2}\right)$ & 14 to $16\left(\mathrm{H}_{2}\right)$ & 299.33 & 283.33 & 272.00 & 244.33 \\
\hline & & Above $16\left(\mathrm{H}_{3}\right)$ & 301.33 & 279.33 & 261.66 & 236.33 \\
\hline & & 12 to $14\left(\mathrm{H}_{1}\right)$ & 330.73 & 326.86 & 316.13 & 305.30 \\
\hline \multirow[t]{3}{*}{$30^{\text {th }}$ Sept $\left(D_{2}\right)$} & Aishwarya $\left(\mathrm{V}_{1}\right)$ & 14 to $16\left(\mathrm{H}_{2}\right)$ & 331.76 & 335.33 & 325.76 & 323.66 \\
\hline & & Above $16\left(\mathrm{H}_{3}\right)$ & 330.53 & 329.60 & 322.60 & 320.13 \\
\hline & & 12 to $14\left(\mathrm{H}_{1}\right)$ & 297.66 & 296.33 & 291.13 & 277.66 \\
\hline \multirow[t]{3}{*}{$30^{\text {th }}$ Sept $\left(D_{2}\right)$} & Sadhana $\left(V_{2}\right)$ & 14 to $16\left(\mathrm{H}_{2}\right)$ & 298.66 & 293.66 & 290.66 & 281.66 \\
\hline & & Above $16\left(\mathrm{H}_{3}\right)$ & 299.66 & 294.33 & 286.66 & 282.66 \\
\hline & & 12 to $14\left(\mathrm{H}_{1}\right)$ & 299.33 & 245.86 & 182.06 & 173.3 \\
\hline \multirow[t]{3}{*}{$15^{\text {th }}$ Oct $\left(D_{3}\right)$} & Aishwarya $\left(\mathrm{V}_{1}\right)$ & 14 to $16\left(\mathrm{H}_{2}\right)$ & 301.00 & 313.53 & 262.50 & 263.03 \\
\hline & & Above $16\left(\mathrm{H}_{3}\right)$ & 297.96 & 240.36 & 218.13 & 280.73 \\
\hline & & 12 to $14\left(\mathrm{H}_{1}\right)$ & 304.66 & 293.00 & 283.00 & 280.00 \\
\hline \multirow[t]{2}{*}{$15^{\text {th }}$ Oct $\left(D_{3}\right)$} & Sadhana $\left(V_{2}\right)$ & 14 to $16\left(\mathrm{H}_{2}\right)$ & 306.66 & 288.33 & 279.33 & 255.96 \\
\hline & & Above $16\left(\mathrm{H}_{3}\right)$ & 304.33 & 285.00 & 278.73 & 185.70 \\
\hline Sem \pm & & & 2.72 & 3.32 & 3.01 & 2.77 \\
\hline$C D(P=0.05)$ & & & NS & 9.52 & 8.65 & 7.96 \\
\hline
\end{tabular}


$\left(\mathrm{D}_{3} \mathrm{~V}_{1} \mathrm{H}_{1}\right)$ which developed $1.33 \%$ yellowing at the end of storage period.

Broccoli sown at $15^{\text {th }}$ September $D_{1}$ had the maximum value of yellowing on the last day of storage and variety Aishwarya had more yellowing percentage than Sadhana. Small diameter broccoli had the least yellowing percentage at the end of storage while the best interaction effect was seen in Aishwarya sown at $30^{\text {th }}$ September with 14 to $16 \mathrm{~cm}$ head $\mathrm{D}_{2} \mathrm{~V}_{1} \mathrm{H}_{2}$ Sadhana sown at $30^{\text {th }}$ September with 12 to $14 \mathrm{~cm}\left(\mathrm{D}_{2} \mathrm{~V}_{2} \mathrm{H}_{1}\right)$. The decrease in chlorophyll due to loss of chlorophyll by retarding chlorophyllase activity was supported with the finding of Yan and Liu (2012). Schoutena et al. (2009) evaluated that for a number of broccoli heads, colour synthesis was larger than the colour decay during

Table 5: Effect of head diameter, variety, date of sowing and their interaction on yellowing (\%) in refrigerated condition.

\begin{tabular}{|c|c|c|c|c|c|}
\hline \multirow[t]{2}{*}{ Date of sowing } & \multirow[t]{2}{*}{ Variety } & \multirow[t]{2}{*}{ Head diameter $(\mathrm{cm})$} & \multicolumn{3}{|c|}{ Days in storage } \\
\hline & & & 5 & 10 & 15 \\
\hline \multicolumn{6}{|c|}{ Effect of date of sowing } \\
\hline $15^{\text {th }}$ Sept $\left(D_{1}\right)$ & & & $0.83(3.58)$ & $0.93(1.88)$ & $1.00(3.98)$ \\
\hline $30^{\text {th }}$ Sept $\left(D_{2}\right)$ & & & $0.67(2.89)$ & $0.67(1.63)$ & $5.38(10.70)$ \\
\hline $15^{\text {th }}$ Oct $\left(D_{3}\right)$ & & & * & * & $14.01(16.41)$ \\
\hline Sem \pm & & & 0.17 & 0.06 & 0.26 \\
\hline$C D(P=0.05)$ & & & 0.05 & 0.16 & 0.95 \\
\hline \multicolumn{6}{|l|}{ Effect of variety } \\
\hline & Aishwarya $\left(\mathrm{V}_{1}\right)$ & & $0.55(2.39)$ & $1.67(4.06)$ & $8.04(13.52)$ \\
\hline & Sadhana $\left(\mathrm{V}_{2}\right)$ & & $0.44(1.91)$ & $0.56(1.91)$ & $5.56(7.21)$ \\
\hline Sem \pm & & & 0.05 & 0.05 & 0.20 \\
\hline$C D(P=0.05)$ & & & NS & 0.13 & 0.77 \\
\hline \multicolumn{6}{|c|}{ Effect of head diameter } \\
\hline & & 12 to $14\left(\mathrm{H}_{1}\right)$ & $0.17(0.97)$ & $0.33(1.35)$ & $1.28(4.44)$ \\
\hline & & 14 to $16\left(\mathrm{H}_{2}\right)$ & $0.18(0.99)$ & $0.67(2.63)$ & $2.22(5.78)$ \\
\hline & & Above $16\left(\mathrm{H}_{3}\right)$ & $1.14(1.62)$ & $2.33(4.98)$ & $16.89(20.87)$ \\
\hline Sem \pm & & & 0.06 & 0.06 & 0.26 \\
\hline$C D(P=0.05)$ & & & 0.17 & 0.16 & NS \\
\hline \multicolumn{6}{|c|}{ Effect of interaction } \\
\hline & & 12 to $14\left(\mathrm{H}_{1}\right)$ & $1.03(5.831)$ & $2.00(8.12)$ & $3.03(10.026)$ \\
\hline \multirow[t]{3}{*}{$15^{\text {th }}$ Sept $\left(D_{1}\right)$} & Aishwarya $\left(\mathrm{V}_{1}\right)$ & 14 to $16\left(\mathrm{H}_{2}\right)$ & * & 3.03 (10.02) & 9.96 (18.39) \\
\hline & & Above $16\left(\mathrm{H}_{3}\right)$ & $2.86(9.73)$ & $10.00(18.42)$ & $40.03(39.23)$ \\
\hline & & 12 to $14\left(\mathrm{H}_{1}\right)$ & * & * & * \\
\hline \multirow[t]{3}{*}{$15^{\text {th }}$ Sept $\left(D_{1}\right)$} & Sadhana $\left(V_{2}\right)$ & 14 to $16\left(\mathrm{H}_{2}\right)$ & * & $1.0(5.73))$ & $13.33(21.32)$ \\
\hline & & Above $16\left(\mathrm{H}_{3}\right)$ & * & $4.00(11.47)$ & $35.00(39.22)$ \\
\hline & & 12 to $14\left(\mathrm{H}_{1}\right)$ & * & * & $1.66(7.33)$ \\
\hline \multirow[t]{3}{*}{$30^{\text {th }}$ Sept $\left(D_{2}\right)$} & Aishwarya $\left(\mathrm{V}_{1}\right)$ & 14 to $16\left(\mathrm{H}_{2}\right)$ & * & * & 4.33 (11.99) \\
\hline & & Above $16\left(\mathrm{H}_{3}\right)$ & * & * & * \\
\hline & & 12 to $14\left(\mathrm{H}_{1}\right)$ & * & * & $1.66(7.33)$ \\
\hline \multirow[t]{3}{*}{$30^{\text {th }}$ Sept $\left(D_{2}\right)$} & Sadhana $\left(\mathrm{V}_{2}\right)$ & 14 to $16\left(\mathrm{H}_{2}\right)$ & * & * & * \\
\hline & & Above $16\left(\mathrm{H}_{3}\right)$ & * & * & $1.67(7.33)$ \\
\hline & & 12 to $14\left(\mathrm{H}_{1}\right)$ & * & * & $1.33(6.53)$ \\
\hline \multirow[t]{3}{*}{$15^{\text {th }}$ Oct $\left(\mathrm{D}_{3}\right)$} & Aishwarya $\left(\mathrm{V}_{1}\right)$ & 14 to $16\left(\mathrm{H}_{2}\right)$ & * & * & * \\
\hline & & Above $16\left(\mathrm{H}_{3}\right)$ & * & * & $7.33(15.69)$ \\
\hline & & 12 to $14\left(\mathrm{H}_{1}\right)$ & * & * & * \\
\hline \multirow[t]{2}{*}{$15^{\text {th }}$ Oct $\left(D_{3}\right)$} & Sadhana $\left(\mathrm{V}_{2}\right)$ & 14 to $16\left(\mathrm{H}_{2}\right)$ & * & * & * \\
\hline & & Above $16\left(\mathrm{H}_{3}\right)$ & * & * & * \\
\hline Sem \pm & & & 0.06 & 0.14 & 0.64 \\
\hline$C D(P=0.05)$ & & & 0.41 & 0.40 & 1.86 \\
\hline
\end{tabular}


the storage period especially at $5^{\circ} \mathrm{C}$ in modified atmosphere which explained green colour is some treatments. Increase in yellowing colour score in packed broccoli during storage was supported by Beer and Crouch (2013).

\section{CONCLUSION}

Studying the various interaction effects of date of planting, variety and head diameter for harvest, it was shown that broccoli sown early i.e. $15^{\text {th }}$ September gave quality in variety Sadhana with the smallest head diameter i.e., 12 to $14 \mathrm{~cm}$ since it possesses the most desirable criteria for marketability. The most common problem in post harvest management of broccoli was yellowing and it was shown clearly that yellowed broccoli still has appreciable amount of ascorbic and TSS content so it can be recommended for consumption even though there is less acceptance in market due to unawareness about the nutrient content.

\section{REFERENCES}

A.O.A.C. (1990). Official Methods of Analysis. $15^{\text {th }}$ Edn., Association of Official Methods of Analytical Chemists, Washington, DC., Arlington, Virginia, USA., ISBN: 0935584-42-0.

Beer, T. de and Crouch, E.M. (2013). Packaging in the Maintenance of Postharvest Keeping Quality of 'Parthenon' Broccoli during Long Term Storage and Subsequent Shelf-Life. Acta Horticulturae. 1007: 65-72

Cantwell, M. and Suslow, T. (1999). Broccoli: Recommendations for maintaining post harvest quality. http://postharvest. ucdavis.edu/produce/producefacts/veg/broccoli.html.

Carvalho, P.T. and Clemente, E. (2004). The influence of the broccoli (Brassica oleracea var. Italic) fill weight on postharvest quality. Ciencia e Tecnologia de Alimentos. 24: 646-651.

Clarke, S.F., Jameson, P.E. and Downs, C. (1994). The influence of 6-benzylaminopurine on post-harvest senescence of floral tissues of broccoli (Brassica oleracea var. italica). Plant Growth Regulation. 14(1): 21-27.

Corcuff, R., Arul, J., Hamza, F., Castaigne, F. and Makhlouf, J. (1996). Storage of broccoli florets in ethanol vapor enriched atmospheres. Postharvest Biology and Technology. 7: 219-229.

Galvao, H.L., Finger, F.L., Puiatti, M., Correa, P.C. and Oliveira, L.S. de. (2008). Effect of pre-cooling and PVC film on the post harvest preservation of broccoli. Revista Brasileira de Armazenamento. 33(2): 101-06.

Ihl, M., Conejeros, E., Mera, M. and Bifani, V. (2001). Quality changes of two sugar snap pea cultivars during cold storage. Acta Horticulturae. 553(2): 749-751.

Jacobsson, A., Nielsen, T., Sjöholm, I. and Wendin, K. (2004). Influence of packaging material and storage condition on sensory quality of broccoli. Postharvest Biology and Technology. 15: 301-310.

Mahfuzah, W.I. Wan, Zulkifli, M.S., Latifah, M.N. and Fauziah, O. (2013). Evaluations for Effectiveness of Calcium Chloride Treatment on the Postharvest Quality of Strawberry. Proc. $7^{\text {th }}$ International Postharvest Symposium. Acta Horticlulturae. 1(1012): 515 .
Nath, A. Bagchi, B., Misra L.K., Bidyut C. Deka, (2011). Changes in post-harvest phytochemical qualities of broccoli florets during ambient and refrigerated storage. Food Chemistry. 127: $1510-14$

Pallavi, S., Pravin, P., Durga, S.B. (2020). Evaluation of modified atmospheric packaging (MAP), chemical treatments and low temperature on biochemical and textural attributes of button mushroom (Agaricus bisporus). Asian Journal of Dairy and Food Research. 39(3): 232-238. DOI: 10.18805/ajdfr.DR-1517

Peris Tortajada, M. (2004). Carbohydrates and Starch. In: [Nollet LML (ed.)], Handbook of Food analysis: Physical Characterisation and Nutrient Analysis. $2^{\text {nd }}$ ed. New York, Marcel Dekker. Pp. 383-408.

Pragya, A., Nisha, P., Kanti, T. and Aashish, D. (2020). Effects of perforations mediated-modified atmosphere packaging (MAP) on shelf life and quality of calcium chloride treated bell pepper (Capsicum annum). Asian Journal of Diary and Food Research. 39(4): 354-358. DOI: 10.18805 lajdfr.DR-200.

Rai, D.R., Tyagi, S.K., Jha, S.N. and Mohan, S. (2008). Qualitative changes in the broccoli (Brassica oleracea italica) under modified atmosphere packaging in perforated polymeric film. Journal of Food Science and Technology. 45(3): 247-250.

Ranganna, S. (1986). Handbook of Analysis and Quality Control for Fruits and Vegetables Product, $2^{\text {nd }}$ Edition (Tata McGraw Hill Publication Co. Ltd, New Delhi). P.112.

Roni, M.S., Mohammad Zakaria, Hossain, M.M., Rasul, M.G. and Siddiqui, M.N. (2014). Effect of temperature on shelf life and ascorbic acid content of broccoli produced with different combinations of nitrogen level and spacing. International Journal of Biosciences. 5(6): 81-86

Schoutena, R.E., Xiaobing, Z., Jan, A., Verschoor, E.C.O., Tijskens, L.M.M. and Olaf van, K. (2009). Development of colour of broccoli heads as affected by controlled atmosphere storage and temperature. Postharvest Biology and Technology. 51: 27-35

Serrano, M., Martinez Romero, D., Guillen, F., Castillo, S. and Valero, D. (2006). Maintenance of broccoli quality and functional properties during cold storage as affected by modified atmosphere packaging. Postharvest Biology and Technology. 39: 61-68.

Starzynska, A., Leja, M. and Mareczek, A. (2003). Physiological changes in the antioxidant system of broccoli flower buds senescing during short-term storage, related to packaging and packaging. Plant Science. 165: 1387-95.

Yan, L. and Liu, S. (2012). Effect of different pre-cooling, packaging and cold storage treatments on quality of broccoli. Acta Horticulturae. 934: 1149-1157. 